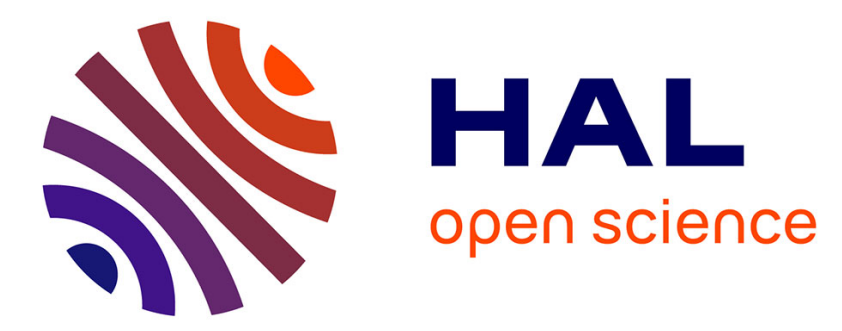

\title{
Time-resolved scanning tomography PIV measurements around a flapping wing
}

Laurent David, Thierry Jardin, Patrick Braud, Alain Farcy

\section{To cite this version:}

Laurent David, Thierry Jardin, Patrick Braud, Alain Farcy. Time-resolved scanning tomography PIV measurements around a flapping wing. Experiments in Fluids, 2012, vol. 52 (n $\left.{ }^{\circ} 4\right)$, pp. 857-864. 10.1007/s00348-011-1148-5 . hal-01132038

\section{HAL Id: hal-01132038 \\ https://hal.science/hal-01132038}

Submitted on 16 Mar 2015

HAL is a multi-disciplinary open access archive for the deposit and dissemination of scientific research documents, whether they are published or not. The documents may come from teaching and research institutions in France or abroad, or from public or private research centers.
L'archive ouverte pluridisciplinaire HAL, est destinée au dépôt et à la diffusion de documents scientifiques de niveau recherche, publiés ou non, émanant des établissements d'enseignement et de recherche français ou étrangers, des laboratoires publics ou privés. 


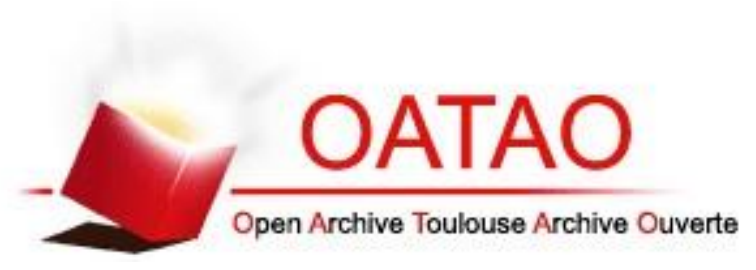

\section{Open Archive Toulouse Archive Ouverte (OATAO)}

OATAO is an open access repository that collects the work of Toulouse researchers and makes it freely available over the web where possible.

This is an author-deposited version published in: http://oatao.univ-toulouse.fr/ Eprints ID: 13653

To link to this article: DOI:10.1007/s00348-011-1148-5

URL: http://dx.doi.org/10.1007/s00348-011-1148-5

To cite this version: David, Laurent and Jardin, Thierry and Braud, Patrick and Farcy, Alain Time-resolved scanning tomography PIV measurements around a flapping wing. (2012) Experiments in Fluids, vol. $52\left(\mathrm{n}^{\circ} 4\right)$. pp. 857-864. ISSN 0723-4864

Any correspondence concerning this service should be sent to the repository administrator: staff-oatao@inp-toulouse.fr 


\title{
Time-resolved scanning tomography PIV measurements around a flapping wing
}

\author{
L. David $\cdot$ T. Jardin $\cdot$ P. Braud $\cdot$ A. Farcy
}

\begin{abstract}
The three-dimensional flow that develops around a finite flapping wing is investigated using a tomographic scanning PIV technique. The acquisition and correlation processes employed to achieve such measurements have been carefully validated. Issues regarding the relevant timescales of the flow and the spanwise spaceresolution are addressed. Results obtained on a hovering flapping wing whose plunging phase is described by a rectilinear motion highlight the influence of the free end condition and the formation of the tip vortex on the leading edge vortices behavior, wing/wake interactions, and wake stabilization.
\end{abstract}

\section{Introduction}

Flapping wings have been of interest to biologists for years and are currently under much consideration by the aeronautical community, due to the recent advent of micro-air vehicles. The problematic associated with such wing concept relies on the comprehension of low Reynolds number unsteady aerodynamic phenomena. Previous studies revealed that mechanisms such as the presence of a leading edge vortex (LEV), the Kramer effect, or the wing/wake interactions are responsible for the generation of a strong lifting force (Dickinson et al. 1999).

Despite the fact that two-dimensional configurations bring interesting insight into the aerodynamics of flapping

L. David $(\bowtie) \cdot$ T. Jardin · P. Braud · A. Farcy

Département Fluides, Thermiques, Combustion, Institut Pprime, CNRS-Université de Poitiers-ENSMA, UPR3346, SP2MI,

Bd Marie et Pierre Curie, téléport 2, 86962, Futuroscope, Chasseneuil, France

e-mail: Laurent.David@univ-poitiers.fr wings (Wang 2000; Kurtulus et al. 2008; Bos et al. 2008; Jardin et al. 2009), the latter is particularly sensitive to the presence of a three-dimensional component. Extended works (Maxworthy 1979; van den Berg and Ellington 1997; Poelma et al. 2006) suggested that spanwise velocities might indeed have an effect on the stability of the LEV. Nevertheless, most researches focused on revolving wing configurations for which the distinction between the effects due to the inertial forces (Coriolis and centrifugal) and that due to the wing tip vortex is delicate. In this paper, we focus on a configuration for which the translation phase is rectilinear, which allows isolating the role of the tip vortex on global flow dynamics.

Visualizing and quantifying the three-dimensional flow that develops around a flapping wing is an essential step toward understanding the influence of three-dimensional effects on the wing/wake interactions and the leading edge vortex behavior. However, few studies reported experimental procedure dedicated to reconstructing an instantaneous three-dimensional representation of the flow, principally through the concatenation of phase-averaged adjacent PIV-2C/3C measurements (Poelma et al. 2006; Ozen and Rockwell 2010; Suryadi et al. 2010). Such technique requires a large amount of runs, which may stand as a limitation for an accurate spanwise resolution. Here, we bypass this issue by directly measuring the instantaneous time-resolved three-dimensional flow field using a scanning PIV technique.

The goal of this paper is essentially twofold. First, we aim at providing a technical description of the scanning PIV-3D3C procedure used to measure large instantaneous flow volumes. The issues associated with the relevant timescales and spanwise discretization of the flow are addressed (in order to ensure time-resolved measurements and spanwise space accuracy, respectively). Second, we 

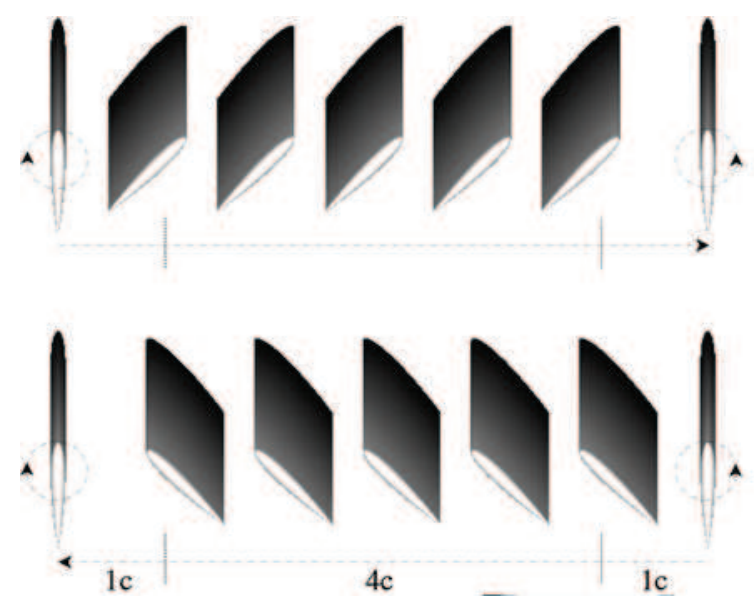

Fig. 1 Description of the symmetric flapping motion

investigate the influence of the free end condition on the development of the leading edge vortex and the wing/wake interactions. We show that the formation of a wing tip vortex promotes the establishment of a three-dimensional stability that considerably reduces wing/wake interactions near the free end. Besides, the flow along most of the wing span is dominated by a two-dimensional instability and highly resembles the one observed in strictly two-dimensional configurations.

\section{Experimental setup}

\subsection{Wing motion}

We are concerned about the flapping motion of a NACA0012 airfoil at a Reynolds number of 1,000. The flapping amplitude is set to 6 chords. The airfoil undergoes a constant speed translation at $45^{\circ}$ fixed angle of attack along 4 chords, after which it is subjected to both rotating and decelerating or accelerating motions whether it, respectively, ends or starts the upstroke/downstroke phases (Fig. 1). The rotating $\Delta \tau_{\mathrm{r}}$ and accelerating/decelerating $\Delta \tau_{t}$ times are set to $c \pi / V_{0}$, where $\mathrm{c}$ and $V_{0}$ are the chord and the constant translation speed of the airfoil, respectively. $V_{0}$ is defined according to the Reynolds number $R e=\mathrm{V}_{0} / c v$, with $v$ being the fluid kinematic viscosity. The flapping period is computed using the following equation: $T=4 c(2+\pi / 2) / V_{0}$.

\subsection{Experimental facilities}

The experiments are conducted in a $1 \times 1 \times 2 \mathrm{~m}^{3}$ water tank filled with $10-\mu \mathrm{m}$ diameter hollow silvered glass particles. Ten grams is introduced, corresponding to a number of particles $N_{\mathrm{p}}=14 \times 10^{9}$ and to a concentration of particles $C_{\mathrm{p}} \approx 9 \times 10^{9} \mathrm{p} / \mathrm{m}^{3}$. The airfoil is a transparent
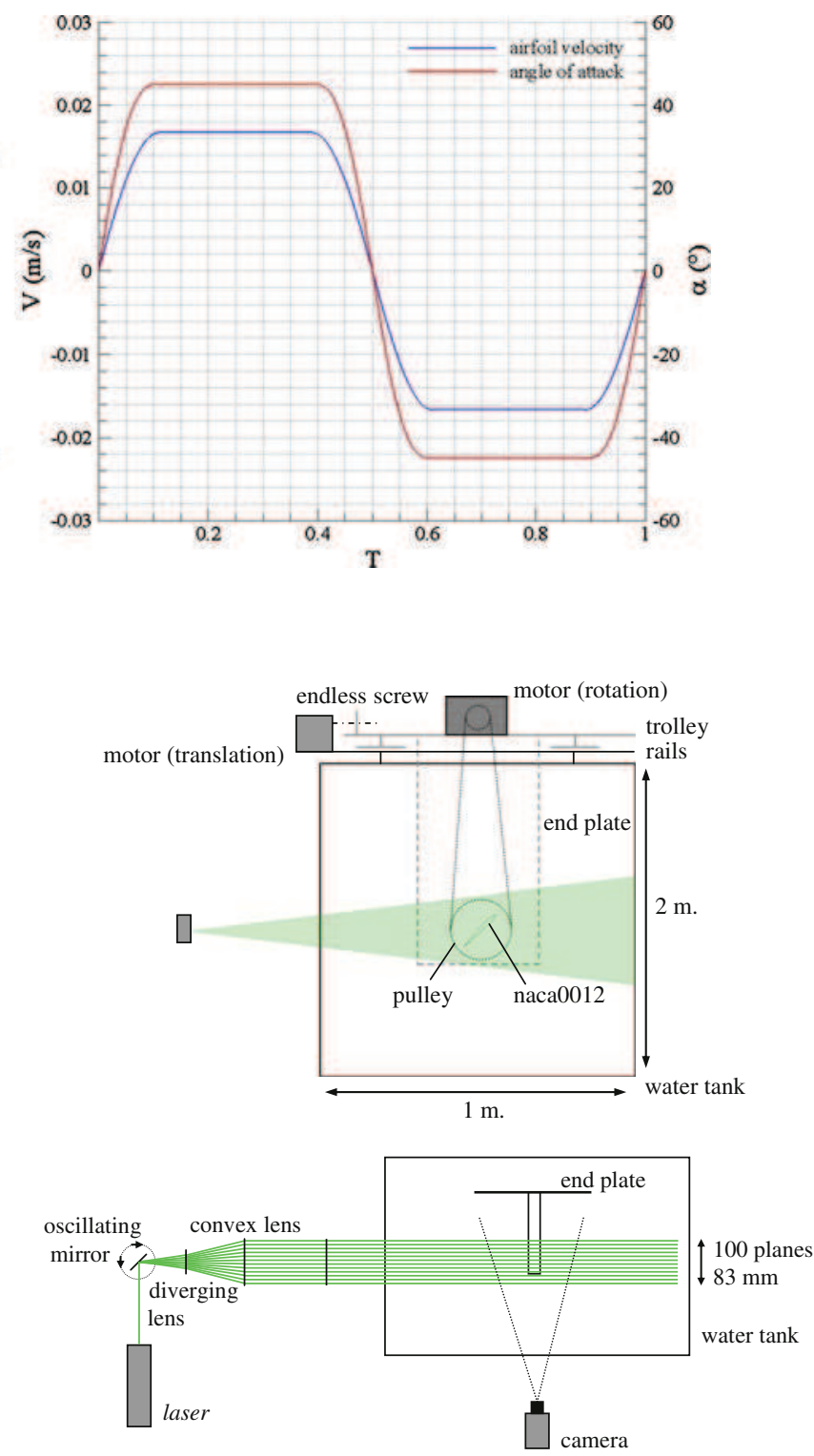

Fig. 2 Experimental setup and scanning device. Front ( $X Y$ plane) and upper ( $Z X$ plane) views

resin NACA0012 profile connected at one end to a Plexiglas plate, the other end being free (Fig. 2). The translating and rotating motions of the airfoil are driven separately through the use of two servo-controlled motors. Their respective mechanical transmissions are achieved by means of an endless screw and a pulley. The aspect ratio $A R=L / c=4$ (where $L$ is the wingspan) has been studied. For the sake of completeness, we give the following dimensions: $c=0.06 \mathrm{~m}, L=0.24 \mathrm{~m}$, $V_{0}=0.017 \mathrm{~m} / \mathrm{s}$, and $T=51.4 \mathrm{~s}$.

\subsection{Scanning PIV-3D3C}

Lately, several techniques have been proposed to simultaneously measure the three velocity components inside a 
volume of the flow, such as algebraic reconstruction tomography (Elsinga et al. 2006) or holography (Arroyo and Hinsch 2008). The former requires multiple recording devices and restricts the measurements to a narrow laser sheet (few centimeters). Holography allows a thicker laser sheet but is not adapted to the measurement of large flow fields involving a great number of particles. In this work, the flow is investigated using a scanning technique rather similar to that described by Kent (1994), Brücker (1995), Hori and Sakakibara (2004) and Fincham (2003).

The two first authors calculated 2D velocity components by PTV or PIV in each laser sheet planes. Afterward, they applied the continuity equation from a symmetrical plane where the out-of-plane velocity component is equal to zero for deducing the three components of the velocity in the volume. Hori and Sakakibara (2004) directly evaluated the three components of velocity for every plane by stereoscopy PIV. Finally, Fincham (2003) directly deduced from 3D-cross-correlation technique the 3 components of velocity for the whole volume of a linearly stratified fluid. Tomography relies in general on the correlation of threedimensional particles mapping, i.e., fields of voxels. In the particular case of high-speed scanning tomography, threedimensional particles mapping is obtained by the highfrequency repetition of adjacent planar recordings. When unsteady flows are considered, one should ensure that the relevant frequencies of the flow may be considered negligible with respect to the scanning frequency. Considering actual tools, this issue limits such measurements to low Reynolds number flows.

The volume is illuminated by means of a $2 \times 20 \mathrm{~mJ}$ Quantronix Darwin-Duo Nd:YLF laser combined with an oscillating mirror. Its depth is limited to $83 \mathrm{~mm}$ by the focal depth of the high-speed camera. The volume is discretized into 100 rigorously parallel and equidistant planes over a time interval of $25 \mathrm{~ms}$, which corresponds to a 4-kHz laser pulse frequency (Fig. 2).

The mirror oscillation is imposed by a low-frequency generator that delivers a periodic and asymmetric triangular signal of amplitude $12.5 \mathrm{~V}$ and frequency $10 \mathrm{~Hz}$, discretized over 1,000 points. Note that the signal is asymmetric to ensure a finite time of $100 \mathrm{~ms}$ between each volume acquisition for the cross-correlation. Thus, the back and forth motions of the mirror, respectively, lasts 34 and $66 \mathrm{~ms}$. Besides, the mechanical oscillation of the mirror generates inertial effects that alter the linearity of the laser sheet motion. Therefore, measurements should be taken during linear phases of the oscillation such that the equidistance between adjacent planes is ensured. This remark implies that the mirror oscillation covers a deeper volume ( $\approx 100 \mathrm{~mm}$ ) than is effectively measured. The red Dirac peaks in Fig. 3 mark the beginning of the linear phases, and Fig. 4 shows the projection of the laser sheet in a $Y Z$ plane recorded by a second camera to adjust the linear part of the total laser sheet displacement. In the same way, the reproducibility of the laser sheet location has been tested

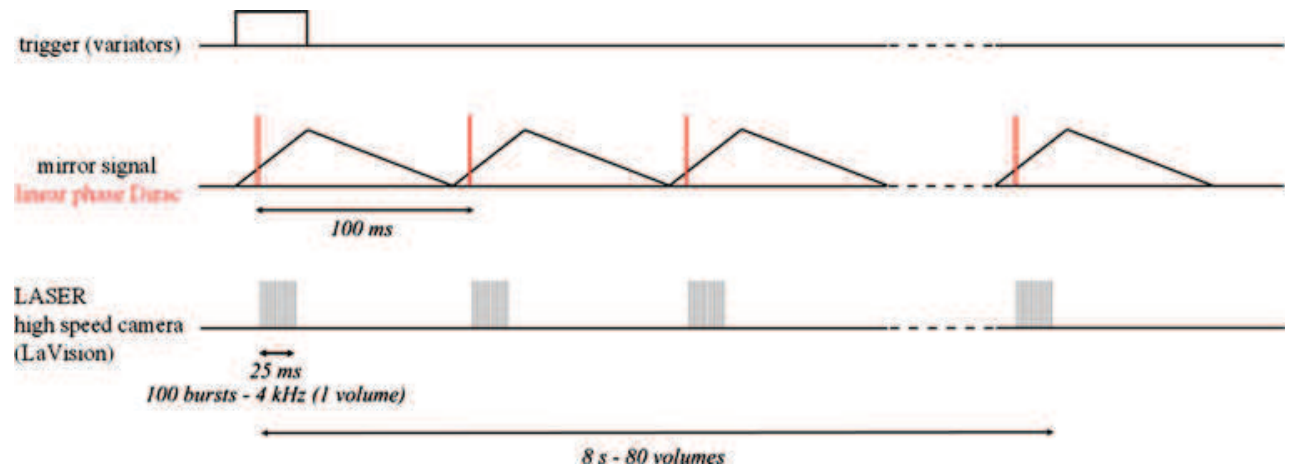

Fig. 3 Time chart for signals and operation

Fig. 4 Laser sheet projection in the $Y Z$ plane for selecting the linear part (for reasons of clearness, one position on five is posted)

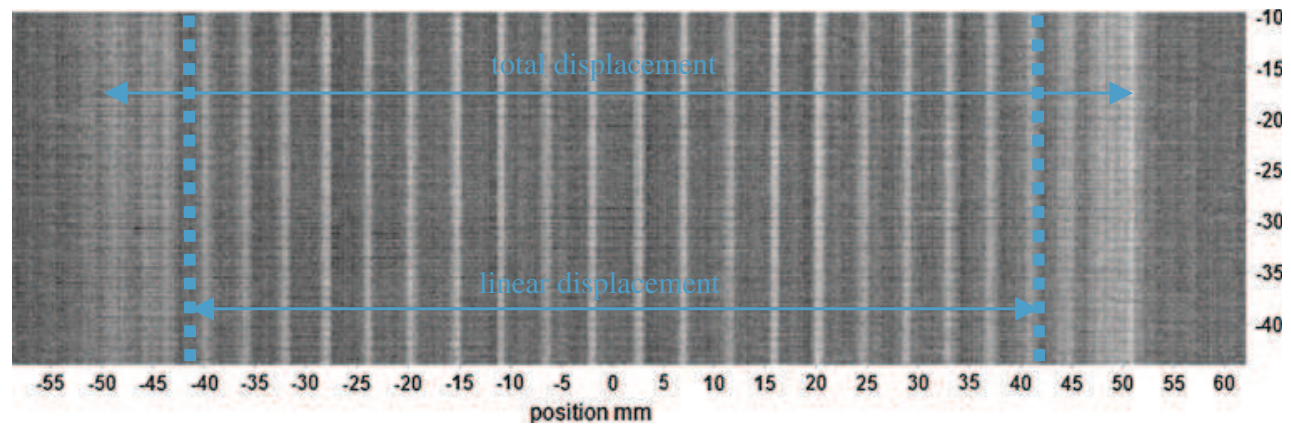


with 100 successive recordings for the same time during the oscillation, and no variation has been detected.

The laser beam interacts with a series of lenses: (1) a diverging lens that emphasizes the oscillation angle, (2) a 145-mm convex lens that reorients the laser beam to ensure parallelism between laser sheets, and (3) a cylinder lens that spreads the laser beam out to form a laser sheet of $0.8 \mathrm{~mm}$ thickness.

A 3D calibration is carried out for different locations of a flat plate target and allows defining a linear model to transform voxel values in physical values not only for the displacement but also for the location of the vectors. Using Davis 7.2 (LaVision) software, the laser is synchronized with a high-speed Photron camera equipped with a 10 bits sensor of resolution $1,024 \times 1,024$ pixels. The camera acquires 100 particle images at a frequency of $4 \mathrm{kHz}$ every $100 \mathrm{~ms}$. Operating at such frequency imposes several restrictions: (1) the finale resolution is set to $1,024 \times$ 784 pixels, (2) the storage capacity (RAM) of the camera being $8 \mathrm{Go}$, the acquisition time is limited to $8 \mathrm{~s}(80 \times 100$ images), and (3) the weak illumination energy returned to the CCD sensor (due to a short exposure time) imposes a lens aperture of $\mathrm{f} \# 8$ which limits the focal depth and hence fixes the volume depth to $83 \mathrm{~mm}$. Prior to the experiments, the focal depth can be estimated using the following equation (Raffel et al. 2007): $\delta_{z}=2 \mathrm{~F} \# d_{\text {diff }}(M+1) / M^{2}$ with $M$ mean magnification factor and $d_{\text {diff }}$ diffraction diameter $d_{\text {diff }}=2.44 \mathrm{~F} \#(M+1) \lambda$. In this work, theoretically $\delta_{z} \approx 30 \mathrm{~mm}$ nevertheless the focus is realized on more than $50 \mathrm{~mm}$ which implies slightly blurred particle images at both end of the volume. The camera is equipped with a $50-\mathrm{mm}$ focal length lens for a $185 \times 140 \mathrm{~mm}^{2}$ area imaging. Recall that these dimensions vary according to the direction $z, 185 \times 140 \mathrm{~mm}^{2}$ being average values.

Based on the maximum airfoil translation speed $\mathrm{V}_{0}$, we get the following particle displacements: $\Delta x, y$, $z=1.67 \mathrm{~mm}$. This leads to a 8.5-voxel displacement in the streamwise directions $(x, y)$ and to a 2-voxel displacement in the spanwise direction (depth $z$ ). If we consider a spanwise displacement, this implies that a particle located in a laser sheet sh at the instant, $t$ will move to a laser sheet sh \pm 2 at the instant $t+\Delta t$. In other words, given that the volume acquisition frequency is set to $4 \mathrm{kHz}$ ( $T=1 / f=0.25 \mathrm{~ms}$ ), the time step is subjected to an uncertainty of the order of $2 \times 0.25=0.5 \mathrm{~ms}$, corresponding to a displacement uncertainty of less than $0.01 \mathrm{~mm}$. In addition, considering a characteristic timescale of $(c \times \sin (\alpha)) /\left(S t_{\mathrm{d}} \times V_{0}\right)=20 \mathrm{~s}$ with $S t_{\mathrm{d}}=0.124$, $c=0.06 \mathrm{~m}$ and $\alpha=45^{\circ}$ and by estimating that an accurate time resolution can be obtained by discretizing this timescale in 10 instants, the acquisition time $(25 \mathrm{~ms})$ can indeed be considered sufficiently small. Note that the characteristic timescale considered here is related to the vortex-shedding process that occurs on a steadily translating airfoil at the non-dimensional frequency $S t_{\mathrm{d}}$.

The volume dimensions $\left(185 \times 140 \times 83 \mathrm{~mm}^{3}\right)$ and the acquisition time $(8 \mathrm{~s})$ not being sufficient to spatially and temporally describe the whole flapping period, measurements are reiterated at different stroke-phase and spanwise regions. Since the flapping motion is symmetric, measurements are limited to a half-period. Streamwise and spanwise spatial overlaps of, respectively, 20 and $5 \mathrm{~mm}$ are imposed between each region. Similarly, a temporal overlap of $1 \mathrm{~s}$ is imposed. The measurement of the full threedimensional velocity field around the flapping wing requires 4 instant runs $\times 4$ space runs. Note that the position of the camera depends on the stroke-phase and spanwise region that is being measured.

The cross-correlation between two successive volumes is achieved using a $\mathrm{C}++$ code. The data being timeresolved, a sequential process is applied. The relatively basic calculation involves a FFTW 3D correlation using a constant interrogation window size and two passes with a discrete 3D window offset on the final pass with $50 \%$ overlap and 3 points parabolic subpixel approximation. Fixed $32 \times 32 \times 8$ voxels windows are used. Note that the correlation volume is isotropic in the real reference frame.

Three-dimensional vector fields are post-processed using a $3 \times 3 \times 3$ median filter. More specifically, vectors that exceed the 1.2-rms bounds (computed over the $3 \times 3 \times 3$ neighbors) are suppressed and replaced by the median value. At the end of the treatment, a correction of the magnification on the velocity fields is carried out on the application point and the components of velocity vectors (Fig. 5).

A divergence-based approach following the same methodology recently suggested by Atkinson et al. (2011) is applied to estimate the accuracy of the measurements. We get some values of the divergence in the range of 0.002-0.06 pixels/pixels with this procedure that gives a maximal uncertainty of 0.8 pixels on the velocity components for the velocity planes at both extremities of the volume and close to the wing where the shadows and the different reflections of the laser sheet in motion add some spurious vectors.

\section{Results}

Measuring the three-dimensional velocity fields using scanning PIV-3D3C is here particularly complex and challenging due to the dimensions of the total investigated region $\left(370 \times 140 \times 320 \mathrm{~mm}^{3}\right)$ and to its application to a moving airfoil. Two main issues are encountered. First, velocity fields are subjected to a relatively strong noise at both ends of the volumes and in the area where the laser 
Fig. 5 Sketch of the volumetric correlation process $(\Delta t=100 \mathrm{~ms})$

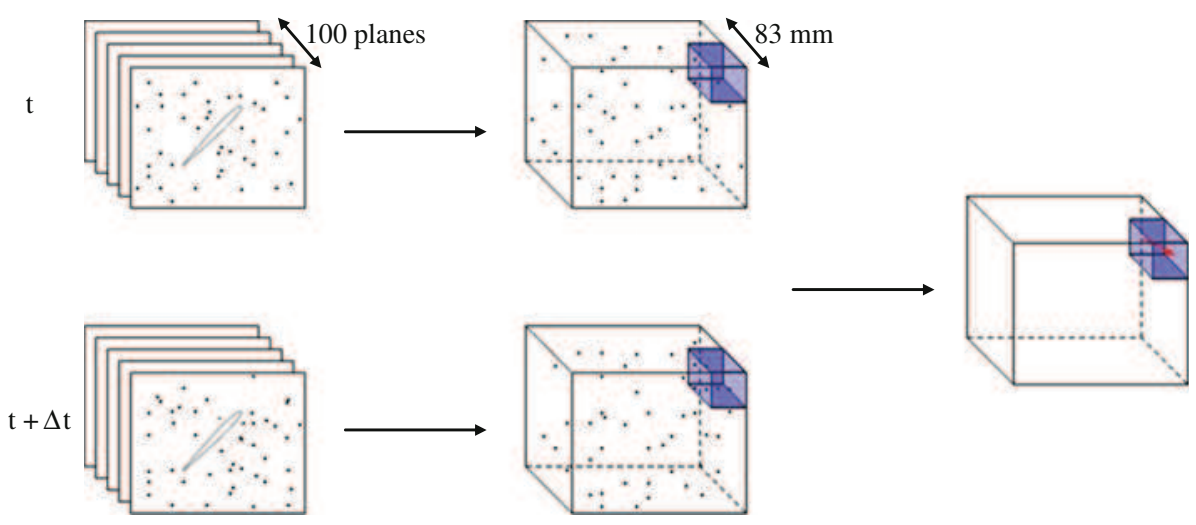

exhibits weak intensity as compared to the prominent LEV1, TEV, and TV1 due to its late formation (during the rotating motion that ends the previous half-stroke). On the contrary, the flow in $p_{\text {root }}$ is characterized by a more perturbed flow. The broad vorticity pattern associated with the leading edge separation depicts the presence of two LEVs (LEV1a and LEV1b), i.e., the occurrence of vortex shedding. The development of such primary instability is not fundamentally altered by the presence of a TV, which allows a more rapid formation of the RV.

Such differences in the wake structure have considerable effects on the development of the following half-stroke flow. At $t=T / 20$, it is shown from frame (b) that the airfoil in $p_{\text {root }}$ interacts with the LEVs and TEV. The latter are responsible for the generation of a fluid jet oriented toward the lower surface of the airfoil and which strengthens the effective incident velocity. This feature adds momentum to the trailing edge shear layer from which develops a starting vortex (SV) whose formation is significantly accelerated. A similar trend is observed for the generation of the leading edge vortex (LEV2). On the contrary, the airfoil in $p_{\text {tip }}$ is not significantly affected by the wing/wake interactions. At this spanwise location, the flow is dominated by the advection of the tip vortex (TV1) under the airfoil lower surface. Note that this downward advection is a result of the classical induced downwash associated with lift production and the formation of the TV.

Frame (c) gives further evidence of the influence of wing/wake interactions on the time evolution of LEV2 and $\mathrm{SV}$. In $p_{\text {root }}$, LEV1b promotes LEV2 stretching from which spots of vorticity are shed and carried downstream. Besides, SV is rapidly shed as LEV1a sweeps along the airfoil lower surface and imparts negative spanwise vorticity at the trailing edge. In $p_{\text {tip }}$, the development of LEV2 is not forced by the wing/wake interactions. Furthermore, the tip downwash decreases the effective angle of attack, hence reducing the production of local vorticity at the leading edge. Both features contribute to the smooth formation of LEV2 near the wing tip. 


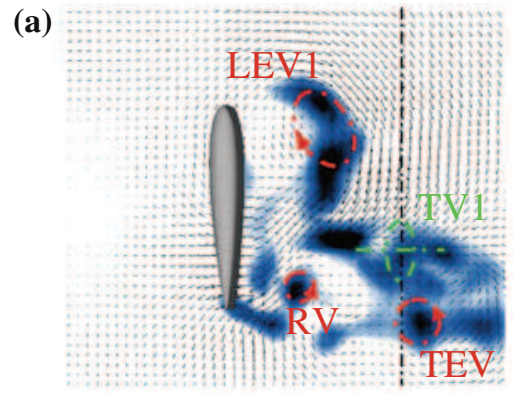

(b)

(c)
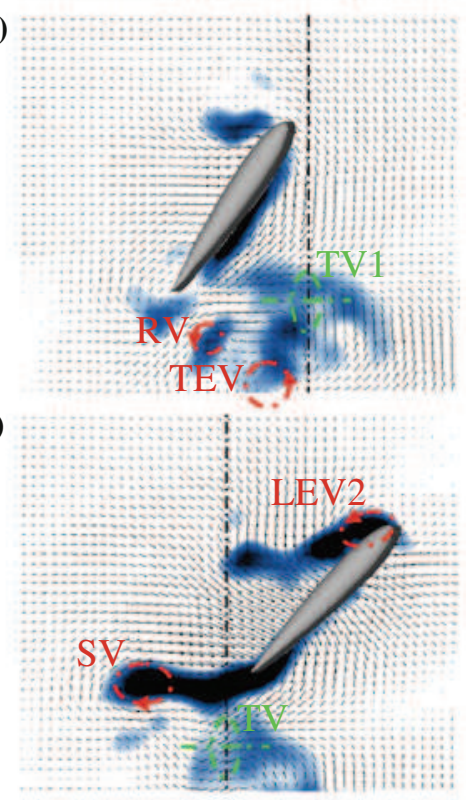
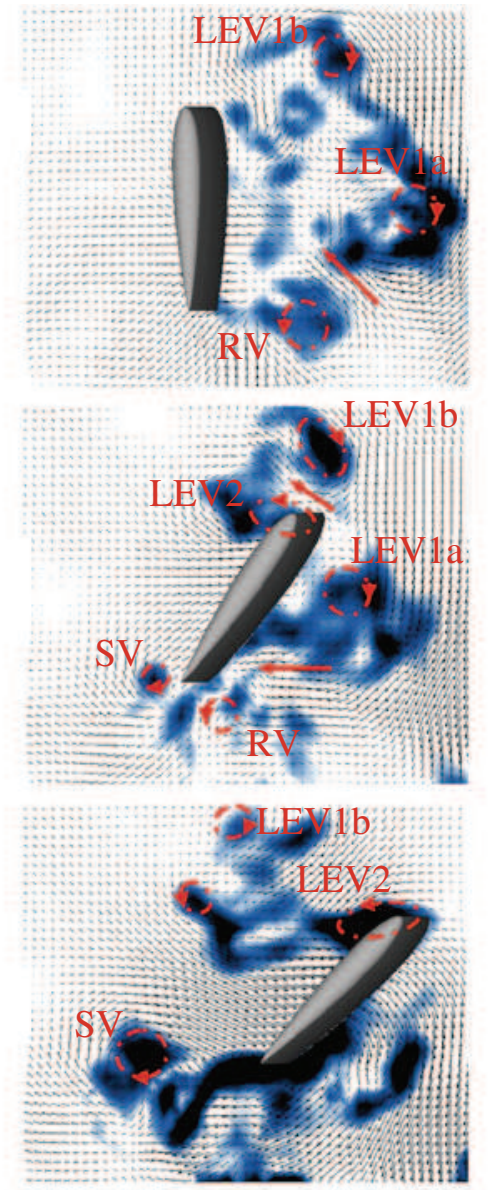
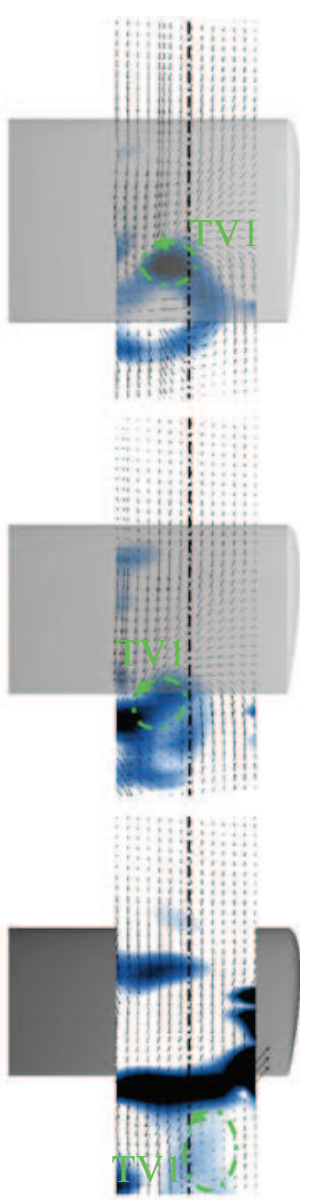

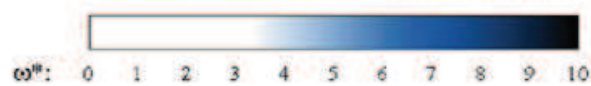

Fig. 6 Velocity vectors and non-dimensional vorticity magnitude contours measured at $t=0(\mathbf{a}), T / 20$ (b) and $2 T / 20$ (c) in two spanwise planes, $p_{\text {tip }} z=0.875 L$ and $p_{\text {root }} z=0.575 L$, and one

\subsection{D instability versus 3D stability}

Figure 7 focuses on the behavior of the leading edge (LEV2) and tip (TV2) vortices at time $t=5 T / 20$ and $t=6 T / 20$, i.e., during the pure translating phase.

At time $t=5 T / 20$, the leading edge vortex in $p_{\text {root }}$ is progressively shed into the wake, leading to the formation of a second LEV2b. The flow converges to a von Karman shedding state characterized by steep variations in aerodynamic loads. Its behavior is dominated by the twodimensional instability, akin to that observed in strictly two-dimensional cases. In $p_{\text {tip }}$, LEV2 is confined at the wing upper surface. More specifically, it grows with the wing translation until it reaches a quasi-steady state. This behavior indicates that the production of spanwise vorticity at the leading edge is balanced by a three-dimensional mechanism arising from the free end condition. At this spanwise location, the flow is dominated by a three- streamwise plane (from left to right). Dashed lines indicate the intersection between the planes in the far left and far right columns

dimensional stability. This particularity is associated with a smooth time evolution of aerodynamic loads. Moreover, the relative position of the LEV and the airfoil upper surface are favorable to the generation of a strong lifting force (the LEV is analogous to a pressure suction region), suggesting that low aspect ratio wings might benefit from three-dimensional effects. Besides, the concomitant development of TV2 along with the translation can be observed. Its position near the trailing edge contributes to its connection with SV, ensuring continuity between streamwise and spanwise vorticities.

Similarly, the flow at time $t=6 T / 20$ exhibits a complex vortical activity in $p_{\text {root }}$ whereas a rather simple organization is observed in $p_{\text {tip. }}$. Such pattern suggests that the near root region will here again be subjected to strong wing/ wake interactions during the subsequent half-stroke, whereas the flow around the wing tip will develop naturally. 


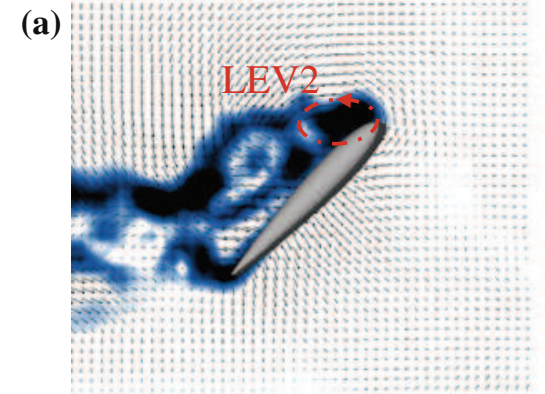

(b)

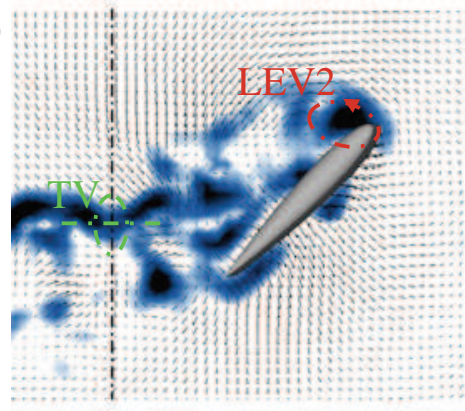

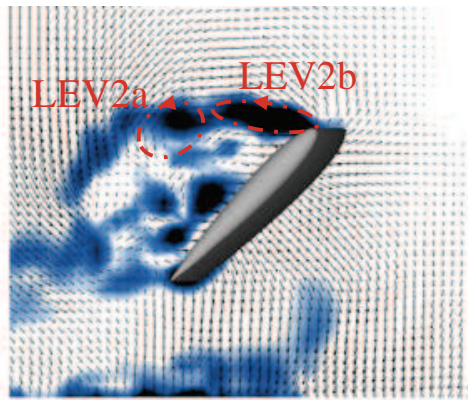

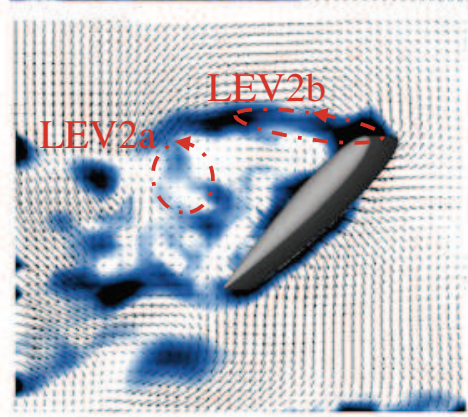

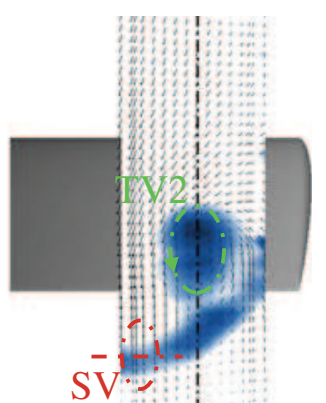

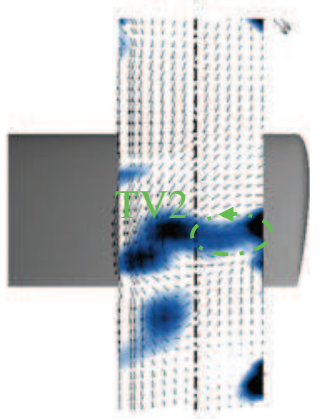

$\omega^{*}:$

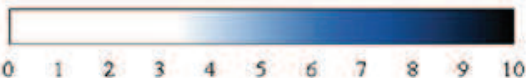

Fig. 7 Velocity vectors and non-dimensional vorticity magnitude contours measured at $t=5 T / 20$ (a) and 6T/20 (b) in two spanwise planes, $p_{\text {tip }} z=0.875 L$ and $p_{\text {root }} z=0.575 L$, and one streamwise plane (from left to right). Dashed lines indicate the intersection

between the planes in the far left and far right columns (no line is depicted in the far left column of frame (a) since the intersection falls outside the represented field)
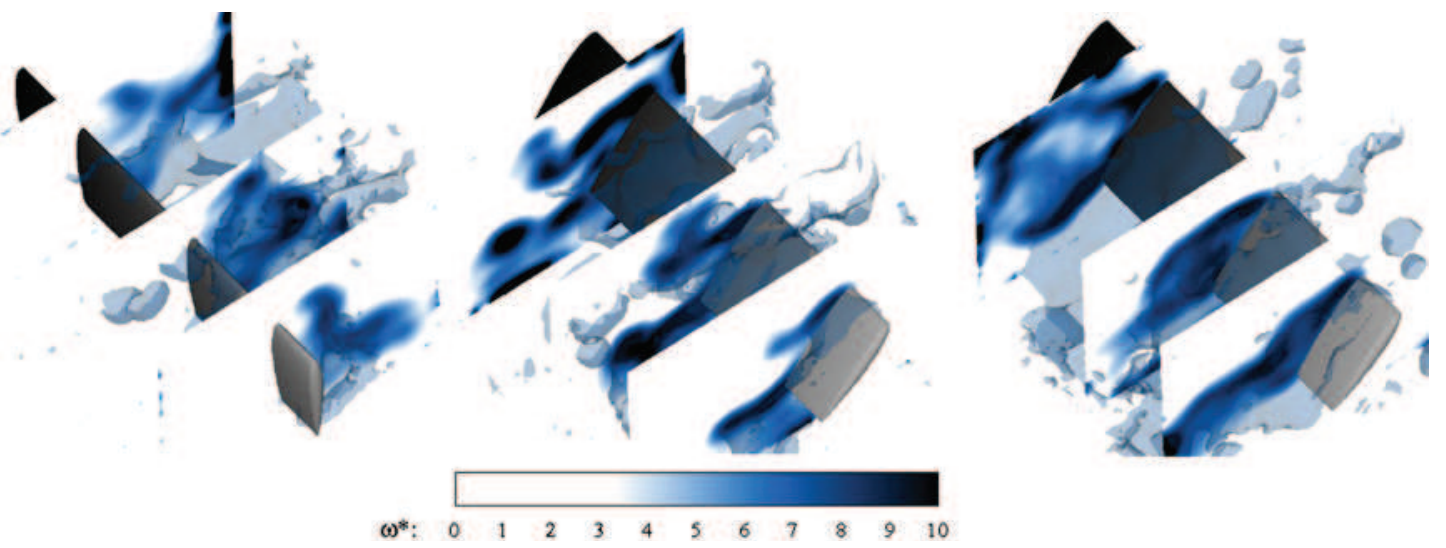

Fig. 8 Vorticity magnitude iso-surfaces in the volume and vorticity magnitude contours in three spanwise planes, respectively, $z=0.2 L$, $z=0.575 \mathrm{~L}$ and $z=0.875 \mathrm{~L}$, measured at $t=0 T / 20,2 T / 20$ and $5 T / 20$ (from left to right)

\subsection{General overview}

Figure 8 provides additional illustrations of the threedimensional flow field obtained at three characteristic instants $t=0 T / 20, t=2 T / 20$, and $t=5 T / 20$. Vorticity iso-surfaces and vorticity contours are displayed in the volume and at $z=0.2 L, z=0.575 L$ and $z=0.875 L$.

The analysis of the three-dimensional flow that develops around a flapping wing whose translation phase is rectilinear (as opposed to revolving wings) demonstrates that the free end condition induces a stabilization of the leading edge vortex near the wing tip. In this region, the tip downwash that originates from the formation of the tip vortex reduces the effective angle of attack, hence the spanwise vorticity production at the leading edge. In addition, the tip vortex might balance the production of spanwise vorticity by promoting the reorientation of spanwise vorticity into streamwise vorticity and advecting it downstream. However, three-dimensional effects only affect the near tip region such that most of the wingspan is 
still dominated by two-dimensional instabilities and, as a consequence, by severe wing/wake interactions. Since three-dimensional effects grow with the formation of the tip vortex, i.e., with the airfoil translation, the frontier between predominantly two-dimensional and threedimensional regions evolves from the wing tip to the wing root. The region swept by this unstable frontier may be qualified as a transition region. It is characterized by both significant wing/wake interactions and three-dimensional stability.

\section{Conclusion}

The measurement of the three velocity components in a volume has been developed to investigate the complex low-speed flow that develops around a flapping wing. The high-speed parallel scanning of a laser sheet synchronized with a high-speed camera allowed particles mapping over a deep volume $(83 \mathrm{~mm})$. Feasibility has been verified by controlling the displacement linearity, the position of the laser sheet in the volume, and ensuring that the scanning timescales were negligible with regard to the relevant timescales of the flow. An adaptive volumetric correlation was employed to extract velocity vectors from the particle recordings. Despite issues related to the fact that distinct flow regions were acquired at different runs, a phenomenological description of the flow was achieved. In particular, the interplay between the tip vortex and the leading edge vortex was characterized. The flow appears to be dominated by two-dimensional instabilities along most of the span and strongly affected by three-dimensional effects near the wing tip.

Acknowledgments This work is funded partially by the 13th CPER and the ANR VIVE3D. Their supports are greatly acknowledged.

\section{References}

Arroyo MP, Hinsch KD (2008) Recent developments of PIV towards 3D measurements. Particle image velocimetry. Top Appl Phys 112:127-154
Atkinson C, Coudert S, Foucaut JM, Stanislas M, Soria J (2011) The accuracy of tomographic particle image velocimetry for measurements of a turbulent boundary layer. Exp Fluids 50:1031-1056

Bos FM, Lentink D, van Oudheusden BW, Bijl H (2008) Influence of wing kinematics on aerodynamic performance in hovering insect flight. J Fluid Mech 594:341-368

Brücker C (1995) Digital-particle-image-velocimetry (DPIV) in a scanning light-sheet: 3D starting flow around a short cylinder. Exp Fluids 19:225-263

Dickinson MH, Lehmann FO, Sane SP (1999) Wing rotation and the aerodynamic basis of insect flight. Science 284:1954-1960

Elsinga GE, Scarano F, Wieneke B, van Oudheusden BW (2006) Tomographic particle image velocimetry. Exp Fluids 41:933-947

Fincham AM (2003) 3 component, volumetric, time-resolved scanning correlation imaging Velocimetry. In: Proceedings of 5th International Symposium on Particle Image Velocimetry, Busan, Korea, pp 2-16

Hori T, Sakakibara J (2004) High speed scanning stereoscopic PIV for 3D vorticity measurement in liquids. Meas Sci Technol 15:1067-1078

Jardin T, David L, Farcy A (2009) Characterization of vortical structures and loads based on time-resolved PIV for asymmetric hovering flapping flight. Exp Fluids 46:847-857

Kent JC (1994) Three-dimensional particle tracking velocimetry: a review. In: Proceedings of Flucome'94, Toulouse, France, pp 309-315

Kurtulus DF, David L, Farcy A, Alemdaroglu N (2008) Aerodynamic characteristics of flapping motion in hover. Exp Fluids 44:23-36

Maxworthy T (1979) Experiments on the Weis-Fogh mechanism of lift generation by insects in hovering flight Part 1 . Dynamics of the 'fling'. J Fluid Mech 93:47-63

Ozen CA, Rockwell D (2010) Vortical structures on a flapping wing. Exp Fluids 50:23-34

Poelma C, Dickson WB, Dickinson MH (2006) Time-resolved reconstruction of the full velocity field around a dynamically scaled flapping wing. Exp Fluids 41:213-225

Raffel M, Willert C, Wereley S, Kompenhans J (2007) Particle image velocimetry-a practical guide, 2nd Edn. Springer, Heidelberg

Suryadi A, Ishii T, Obi S (2010) Stereo PIV measurement of a finite, flapping rigid plate in hovering condition. Exp Fluids 49:447-460

Van den Berg C, Ellington CP (1997) The three-dimensional leadingedge vortex of a 'hovering' model hawkmoth. Philos Trans R Soc Lond B 352:329-340

Wang ZJ (2000) Two-dimensional mechanism for insect hovering. Phys Rev Lett 85:2216-2219 